\title{
The Application of Real Convolution for Analytically Evaluating Fermi-Dirac-Type and Bose-Einstein-Type Integrals
}

\author{
Jerry P. Selvaggi ${ }^{1}{ }^{1}$ and Jerry A. Selvaggi ${ }^{2}$ \\ ${ }^{1}$ Private Practice, Schenectady, NY 12308, USA \\ ${ }^{2}$ Private Practice, Pittsburgh, PA 15217, USA \\ Correspondence should be addressed to Jerry P. Selvaggi; jpsst44@gmail.com
}

Received 13 September 2017; Revised 6 March 2018; Accepted 20 March 2018; Published 6 May 2018

Academic Editor: N. K. Govil

Copyright ( 2018 Jerry P. Selvaggi and Jerry A. Selvaggi. This is an open access article distributed under the Creative Commons Attribution License, which permits unrestricted use, distribution, and reproduction in any medium, provided the original work is properly cited.

\begin{abstract}
The Fermi-Dirac-type or Bose-Einstein-type integrals can be transformed into two convergent real-convolution integrals. The transformation simplifies the integration process and may ultimately produce a complete analytical solution without recourse to any mathematical approximations. The real-convolution integrals can either be directly integrated or be transformed into the Laplace Transform inversion integral in which case the full power of contour integration becomes available. Which method is employed is dependent upon the complexity of the real-convolution integral. A number of examples are introduced which will illustrate the efficacy of the analytical approach.
\end{abstract}

\section{Introduction}

This article presents an extension to the method developed by J. A. Selvaggi and J. P. Selvaggi [1] for analytically evaluating Fermi-Dirac-type and Bose-Einstein-type integrals. The FermiDirac and Bose-Einstein integrals occupy an important role in areas such as solid-state physics and statistical mechanics. There exists numerous approximate analytical methods for evaluating these integrals, but none of the published methods give a general technique for analytically evaluating these integrals valid for the full range of the degeneracy parameter. This article illustrates a general method for attacking these integrals using real convolution.

The right-hand side of (1) and (2) will be defined as Fermi-Dirac-type integral and the Bose-Einstein-type integral, respectively.

$$
\begin{aligned}
& F(\xi)=\int_{0}^{\infty} \frac{f(x)}{1+e^{x-\xi}} d x, \\
& B(\xi)=\int_{0}^{\infty} \frac{f(x)}{-1+e^{x-\xi}} d x .
\end{aligned}
$$

Analytical evaluation of these integrals yields the functions $F(\xi)$ and $B(\xi)$. These functions will be defined as the FermiDirac function and the Bose-Einstein function, respectively. The parameter, $\xi$, is called the degeneracy parameter, a term encountered in statistical mechanics. However, as far as the integrals in (1) and (2) are concerned, $\xi$ represents any real number. In general, integrals of this type do not allow for closed-form solutions in terms of elementary functions. This article introduces a general method for analytically evaluating the integrals given in (1) and (2) for various functions, $f(x)$. The denominator of the integrands in (1) or (2) is exactly that found in the familiar Fermi-Dirac [2-4] or BoseEinstein integrals $[5,6]$. These integrals are often encountered in statistical and quantum statistical mechanics [7-9]. The authors will mainly consider the Fermi-Dirac functions and Bose-Einstein functions within that domain for which $\xi \epsilon$ $\mathbb{R} \geq 0$. If $\xi \in \mathbb{R}<0$, (1) and (2) may be solved by elementary methods.

Numerous techniques have been employed to analytically approximate and numerically evaluate the half-order FermiDirac functions [2, 10-18] and half-order Bose-Einstein functions $[5,17,18]$ (see Appendix). A relatively new representation 
for these integrals, for $f(x)=x^{v} \forall v>-1$, is the Polylogarithm function [19-22]. This function has been studied extensively in the literature. In fact, mathematical software such as Mathematica [23] uses a Polylogarithm algorithm to numerically compute the Fermi-Dirac and Bose-Einstein integrals. However, the authors will illustrate that the application of real convolution allows for the complete analytical evaluation of the integrals in (1) and (2) for a wide range of functions, $f(x)$. The authors have already employed this technique [1] to analytically evaluate the integral in (1) for the well-known and important case of the half-order FermiDirac functions where $f(x)=x^{m-1 / 2} \forall m \in \mathbb{Z}^{\geq}$. A few examples will be considered which will help to illustrate the efficacy of the method. Each solution was numerically checked by employing Mathematica [23] and other numerical algorithms.

\section{Theoretical Development}

Rewrite the integrals given in (1) and (2) in terms of two convergent real-convolution integrals [24]. The observation that real convolution can be employed is the main focus of this article. In fact, once the integrals are transformed into the proper real-convolution form, the difficulty in analytically evaluating (1) and (2) may be substantially reduced.

In order to see that (1) and (2) can each be transformed into two convergent real-convolution integrals requires that each integral be put into the proper form. To this end, rewrite (1) and (2) as follows:

$$
\begin{aligned}
& F(\xi)=\int_{0}^{\xi} \frac{f(x)}{1+e^{-(\xi-x)}} d x+\int_{\xi}^{\infty} \frac{f(x) e^{-(x-\xi)}}{1+e^{-(x-\xi)}} d x, \\
& B(\xi)=-\int_{0}^{\xi} \frac{f(x)}{1-e^{-(\xi-x)}} d x+\int_{\xi}^{\infty} \frac{f(x) e^{-(x-\xi)}}{1-e^{-(x-\xi)}} d x,
\end{aligned}
$$

valid $\forall \xi \in \mathbb{R} \geq 0$.

The denominators in each of the integrals in (3) and (4) can be expanded in a binomial expansion. Each expansion results in a convergent integral within its limits of integration. Employing (3) and expanding the denominator of both integrals in their appropriate binomial expansion yields

$$
\begin{aligned}
F(\xi) & =\int_{0}^{\xi} f(x) d x+\sum_{p=1}^{\infty}(-1)^{p} \\
& \cdot\left[\int_{0}^{\xi} f(x) e^{-p(\xi-x)} d x-\int_{\xi}^{\infty} f(x) e^{-p(x-\xi)} d x\right] .
\end{aligned}
$$

Likewise, (4) can be rewritten as

$$
\begin{aligned}
B(\xi) & =-\int_{0}^{\xi} f(x) d x \\
& -\sum_{p=1}^{\infty}\left[\int_{0}^{\xi} f(x) e^{-p(\xi-x)} d x-\int_{\xi}^{\infty} f(x) e^{-p(x-\xi)} d x\right] .
\end{aligned}
$$

Depending upon the complexity of the function, $f(x)$, direct integration may be possible. However, the authors will develop the necessary mathematical machinery employing the Laplace Transform inversion integral and contour integration of complex variable theory in order to analytically evaluate (5) and (6). The two integrals within the summation in (5), for example, are real-convolution integrals [24] defined as follows:

$$
\begin{aligned}
& \int_{0}^{\xi} f(x) e^{-p(\xi-x)} d x=\frac{1}{2 \pi i} \int_{\sigma-i \infty}^{\sigma+i \infty} \frac{\mathbb{F}(s) e^{\xi s}}{(s+p)} d s, \\
& \int_{\xi}^{\infty} f(x) e^{-p(x-\xi)} d x=\frac{1}{2 \pi i} \int_{\sigma-i \infty}^{\sigma+i \infty} \frac{\mathbb{F}(s) e^{\xi s}}{(-s+p)} d s,
\end{aligned}
$$

where $\mathbb{F}(s)$ is defined as the Laplace Transform of $f(x)$. Both expressions are valid for $\forall \xi \in \mathbb{R}>0$. One can eventually relax the restriction on $\xi$ to include $\forall \xi \in \mathbb{R} \geq 0$. Substituting (7) into (5) yields the following:

$$
\begin{aligned}
F(\xi)= & \int_{0}^{\xi} f(x) d x \\
& +2 \sum_{p=1}^{\infty}(-1)^{p} \frac{1}{2 \pi i} \int_{\sigma-i \infty}^{\sigma+i \infty} \frac{s \mathbb{F}(s) e^{\xi s}}{\left(s^{2}-p^{2}\right)} d s .
\end{aligned}
$$

Equation (8) represents an exact alternative expression for (1). The only restriction put upon $f(x)$ is that it must be a function which allows the integral in (1) to be convergent. Of course, $f(x)$ must have a Laplace Transform. The same procedure illustrated above yields the following expression for the Bose-Einstein-type integral.

$$
B(\xi)=-\int_{0}^{\xi} f(x) d x-2 \sum_{p=1}^{\infty} \frac{1}{2 \pi i} \int_{\sigma-i \infty}^{\sigma+i \infty} \frac{s \mathbb{F}(s) e^{\xi s}}{\left(s^{2}-p^{2}\right)} d s .
$$

The first integral, shown in (8) or (9), may be quite simple for practical problems if $f(x)$ is an analytically integrable function. The second integral, shown in (8) or (9), is the inverse Laplace Transform [24-26] defined as follows:

$$
g(\xi, p)=\mathfrak{Q}^{-1}[G(s)]=\frac{1}{2 \pi i} \int_{\sigma-i \infty}^{\sigma+i \infty} \frac{s \mathbb{E}(s) e^{\xi s}}{\left(s^{2}-p^{2}\right)} d s,
$$

where

$$
G(s)=\frac{s \mathbb{F}(s)}{\left(s^{2}-p^{2}\right)} .
$$

The contour defined by the limits of integration in (10a) is called the Bromwich Contour [24-26]. Of course, there is no guarantee that the analytical evaluation of the inversion integral of (10a) will be an easy task. However, for many practical problems found in the literature, this appears not to be a problem.

\section{Application of Real Convolution}

3.1. Example 1. This simple example is used to verify the method developed in Section 2. Let $f(x)=x^{m} \forall m \in \mathbb{Z}^{2}$ 
and evaluate the expression for the Fermi-Dirac function by employing (8). The result is as follows:

$$
\begin{aligned}
F_{m}(\xi) & \\
= & \frac{\xi^{m+1}}{m+1} \\
& \quad+2(m !) \sum_{p=1}^{\infty}(-1)^{p} \frac{1}{2 \pi i} \int_{\sigma-i \infty}^{\sigma+i \infty} \frac{e^{\xi s}}{s^{m}\left(s^{2}-p^{2}\right)} d s .
\end{aligned}
$$

Evaluate the inversion integral in (E.1.1) by applying the theory of residues of complex variable theory. This is accomplished by choosing the appropriate contour in the complex plane. Figure 1 represents just one possible contour chosen to evaluate the integral in (E.1.1). The contour is closed in the left-hand plane in order to ensure convergence.

Employing the contour of Figure 1 yields the following:

$$
\begin{aligned}
\frac{1}{2 \pi i} \oint \frac{e^{\xi s}}{s^{m}\left(s^{2}-p^{2}\right)} d s \\
=\frac{1}{2 \pi i} \int_{\sigma-i \infty}^{\sigma+i \infty} \frac{e^{\xi s}}{s^{m}\left(s^{2}-p^{2}\right)} d s \\
=\left.\frac{e^{\xi s}}{s^{m}(s-p)}\right|_{s=p e^{\pi i}} \\
\quad+\left.\frac{1}{(m-1) !} \frac{d^{m-1}}{d s^{m-1}}\left(\frac{e^{\xi s}}{s^{2}-p^{2}}\right)\right|_{s=0} \\
=-\frac{e^{-\xi p}}{2 p^{m+1} e^{m \pi i}}+\left.\frac{1}{(m-1) !} \frac{d^{m-1}}{d s^{m-1}}\left(\frac{e^{\xi s}}{s^{2}-p^{2}}\right)\right|_{s=0} .
\end{aligned}
$$

The integral around path 2 for the contour of Figure 1 contributes zero due to Jordan's Lemma [25]. We now have the following expression valid $\forall \xi \in \mathbb{R} \geq 0$ :

$$
\begin{aligned}
& F_{m}(\xi)=\frac{\xi^{m+1}}{m+1}-\sum_{p=1}^{\infty}(-1)^{p} \\
& \quad\left[\frac{m ! e^{-\xi p}}{p^{m+1} e^{m \pi i}}-\left.2 m \frac{d^{m-1}}{d s^{m-1}}\left(\frac{e^{\xi s}}{s^{2}-p^{2}}\right)\right|_{s=0}\right] .
\end{aligned}
$$

Case $1(m=0)$.

$$
F_{0}(\xi)=\xi-\sum_{p=1}^{\infty} \frac{(-1)^{p} e^{-\xi p}}{p}=\ln \left(1+e^{\xi}\right) .
$$

Case $2(m=1)$.

$$
F_{1}(\xi)=\frac{\pi^{2}}{6}+\frac{\xi^{2}}{2}+\sum_{p=1}^{\infty} \frac{(-1)^{p} e^{-\xi p}}{p^{2}} .
$$

Case $3(m=2)$.

$$
F_{2}(\xi)=\frac{\pi^{2} \xi}{3}+\frac{\xi^{3}}{3}-2 \sum_{p=1}^{\infty} \frac{(-1)^{p} e^{-\xi p}}{p^{3}} .
$$

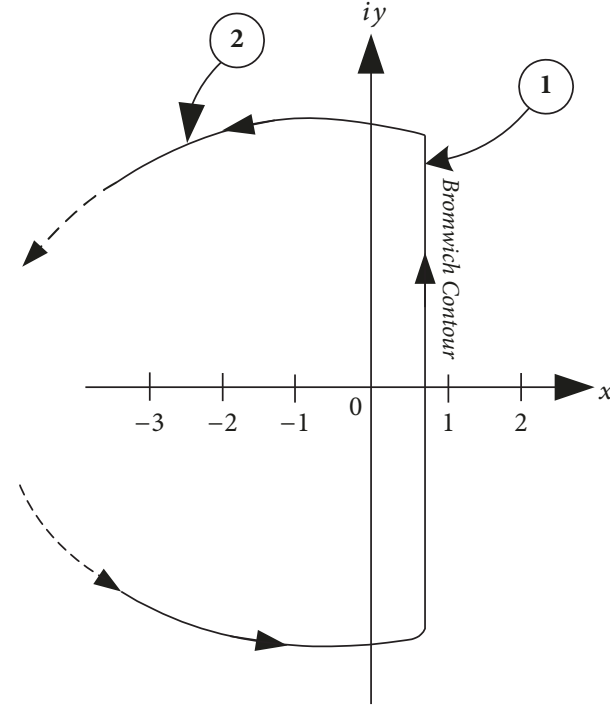

FIgURE 1: Contour used for Examples 1 and 2.

The Fermi-Dirac function, $F_{m}(\xi)$, for any value of $m \in \mathbb{Z}^{\geq}$can easily be evaluated. Employing (5), instead of (8), for $m=0$ yields the following:

$$
\begin{aligned}
& F_{0}(\xi) \\
& \qquad \int_{0}^{\xi} d x \\
& \quad+\sum_{p=1}^{\infty}(-1)^{p}\left[\int_{0}^{\xi} e^{-p(\xi-x)} d x-\int_{\xi}^{\infty} e^{-p(x-\xi)} d x\right] \\
& =\xi+\sum_{p=1}^{\infty} \frac{(-1)^{p}}{p}\left(1-e^{-p \xi}\right)-\sum_{p=1}^{\infty} \frac{(-1)^{p}}{p} \\
& =\ln \left(1+e^{\xi}\right) .
\end{aligned}
$$

Alternatively, (5) can be employed to evaluate $F_{m}(\xi)$ for any $m \in \mathbb{Z}^{\geq}$. However, employing contour integration avails one of all the mathematical machineries of complex variable theory and allows one to tackle a wide range of integrals that may otherwise be rather complicated to evaluate by alternative methods. The same analysis can be employed to analytically evaluate the Bose-Einstein function, $B(\xi)$, for $f(x)=x^{m} \forall m \in \mathbb{Z}^{\geq}$.

3.2. Example 2. As a second example, consider a slightly more complicated function. Let $f(x)=\sin (a x) \forall a \in \mathbb{R}$, and find the expression for the Fermi-Dirac function. Employing (8) and using the contour of Figure 1 yield the following:

$$
\begin{gathered}
F(\xi)=\int_{0}^{\xi} \sin (a x) d x+2 a \sum_{p=1}^{\infty}(-1)^{p} \frac{1}{2 \pi i} \\
\cdot \int_{\sigma-i \infty}^{\sigma+i \infty} \frac{s e^{\xi s}}{\left(s^{2}+a^{2}\right)\left(s^{2}-p^{2}\right)} d s
\end{gathered}
$$




$$
\begin{aligned}
= & \frac{1-\cos (a \xi)}{a}+2 a \sum_{p=1}^{\infty} \frac{(-1)^{p}}{p^{2}+a^{2}} \frac{1}{2 \pi i} \oint\left[\frac{s e^{\xi s}}{\left(s^{2}-p^{2}\right)}\right. \\
& \left.-\frac{s e^{\xi s}}{\left(s^{2}+a^{2}\right)}\right] d s \\
= & \frac{1}{a}-\pi \frac{\cos (\xi a)}{\sinh (\pi a)}+a \sum_{p=1}^{\infty} \frac{(-1)^{p} e^{-p \xi}}{p^{2}+a^{2}} .
\end{aligned}
$$

The same analysis can be used to compute the Bose-Einstein function by employing (6) or (9).

3.3. Example 3. Consider an even more difficult problem. Substituting $f(x)=\sqrt{x}$ into (1) and (2) yields the wellknown half-order Fermi-Dirac function, $F_{1 / 2}(\xi)$, and the half-order Bose-Einstein function, $B_{1 / 2}(\xi)$, respectively (see Appendix). The half-order Fermi-Dirac function has already been analytically evaluated by J. A. Selvaggi and J. P. Selvaggi [1]. Let us attempt to analytically evaluate the half-order BoseEinstein function, $\forall \xi \in \mathbb{R} \geq 0$, and use the result to find an alternative expression for the half-order Fermi-Dirac function not discussed in J. A. Selvaggi and J. P. Selvaggi [1]. The half-order Bose-Einstein function is given by

$$
B_{1 / 2}(\xi)=\int_{0}^{\infty} \frac{\sqrt{x}}{-1+e^{x-\xi}} d x,
$$

valid $\forall \xi \in \mathbb{R} \geq 0$. The Laplace Transform of $\sqrt{x}$ is $\sqrt{\pi} / 2 s^{3 / 2}$. Employ (9) to evaluate the integral in (E.3.1) as follows:

$$
\begin{aligned}
B_{1 / 2}(\xi)= & -\frac{2}{3} \xi^{3 / 2} \\
& -\sqrt{\pi} \sum_{p=1}^{\infty} \frac{1}{2 \pi i} \int_{\sigma-i \infty}^{\sigma+i \infty} \frac{e^{\xi s}}{\sqrt{s}\left(s^{2}-p^{2}\right)} d s .
\end{aligned}
$$

The analytical evaluation of the inversion integral given in (E.3.2) is expedited by employing the contour shown in Figure 2. The authors call this the Fermi-Dirac contour because of its utility in aiding in the analytical evaluation of the half-order Fermi-Dirac functions, $F_{-1 / 2}(\xi)$ and $F_{1 / 2}(\xi)$. Once again, the Fermi-Dirac contour is closed in the lefthand plane in order to ensure convergence.

Employing contour integration in the complex plane and applying the theory of residues yield the following:

$$
\begin{aligned}
\frac{1}{2 \pi i} \oint & \frac{e^{\xi s}}{\sqrt{s}\left(s^{2}-p^{2}\right)} d s \\
= & \frac{1}{2 \pi i} \int_{\sigma-i \infty}^{\sigma+i \infty} \frac{e^{\xi s}}{\sqrt{s}\left(s^{2}-p^{2}\right)} d s \\
& +\frac{1}{2 \pi i} \int_{\infty}^{0} \frac{e^{i \xi y}}{\sqrt{y e^{\pi i / 2}}\left[\left(y e^{\pi i / 2}\right)^{2}-p^{2}\right]} i d y \\
& +\frac{1}{2 \pi i} \int_{0}^{\infty} \frac{e^{i \xi y}}{\sqrt{y e^{-3 \pi i / 2}}\left[\left(y e^{-3 \pi i / 2}\right)^{2}-p^{2}\right]} i d y .
\end{aligned}
$$

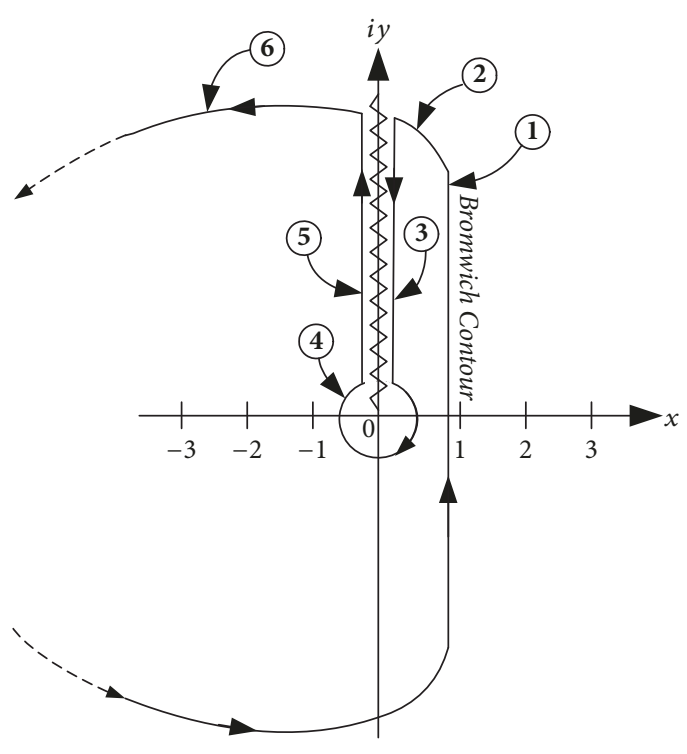

Figure 2: Fermi-Dirac contour.

Only the line integrals along paths 1 (Bromwich Contour), 3, and $\mathbf{5}$ in Figure 2 contribute a nonzero quantity to the closed line integral on the left-hand side of (E.3.3). The closed line integral on the left-hand side of (E.3.3) encloses only simple poles since the branch point at the origin has been excluded from the contour of integration. The mathematical details are found in J. A. Selvaggi and J. P. Selvaggi [1]. The result is as follows:

$$
\begin{aligned}
-\frac{i e^{-p \xi}}{2 p^{3 / 2}}= & \frac{1}{2 \pi i} \int_{\sigma-i \infty}^{\sigma+i \infty} \frac{e^{\xi s}}{\sqrt{s}\left(s^{2}-p^{2}\right)} d s \\
& +\frac{e^{-\pi i / 4}}{2 \pi} \int_{0}^{\infty} \frac{e^{i \xi y}}{\sqrt{y}\left[y^{2}+p^{2}\right]} d y \\
& -\frac{e^{3 \pi i / 4}}{2 \pi} \int_{0}^{\infty} \frac{e^{i \xi y}}{\sqrt{y}\left[y^{2}+p^{2}\right]} d y .
\end{aligned}
$$

After simplifying the algebra, the Inverse Laplace Transform becomes

$$
\begin{aligned}
& \frac{1}{2 \pi i} \int_{\sigma-i \infty}^{\sigma+i \infty} \frac{e^{\xi s}}{\sqrt{s}\left(s^{2}-p^{2}\right)} d s \\
& \quad=-\frac{1}{2 p^{3 / 2}}\left[e^{p \xi} \operatorname{Erfc}(\sqrt{p \xi})+e^{-p \xi} \operatorname{Erfi}(\sqrt{p \xi})\right]
\end{aligned}
$$

Once again, the mathematical details used in obtaining (E.3.5) are given in J. A. Selvaggi and J. P. Selvaggi [1]. Employing (E.3.5) and (E.3.2) yields the following half-order Bose-Einstein function.

$$
\begin{aligned}
& B_{1 / 2}(\xi)=-\frac{2}{3} \xi^{3 / 2}+\frac{\sqrt{\pi}}{2} \sum_{p=1}^{\infty} \frac{1}{p^{3 / 2}} \\
& \cdot\left[e^{p \xi} \operatorname{Erfc}(\sqrt{p \xi})+e^{-p \xi} \operatorname{Erfi}(\sqrt{p \xi})\right] .
\end{aligned}
$$


The functions $\operatorname{Erfc}(\bullet)$ and $\operatorname{Erfi}(\bullet)$ are the Complimentary and Imaginary Error functions, respectively. The evaluation of $B_{1 / 2}(\xi) \forall \xi \in \mathbb{R}<0$ is solved by elementary methods and will not be discussed in this article. The half-order Fermi-Dirac function is found by employing the following duplication formula:

$$
F_{v}(\xi)=B_{v}(\xi)-\frac{1}{2^{v}} B_{v}(2 \xi)
$$

This expression is discussed in Clunie [5] and Dingle [6], and it links the Bose-Einstein functions to the Fermi-Dirac functions. When $f(x)=x^{v}$ and $v=1 / 2$, the following expression is found:

$$
F_{1 / 2}(\xi)=B_{1 / 2}(\xi)-\frac{1}{\sqrt{2}} B_{1 / 2}(2 \xi)
$$

Employing (E.3.8) and (E.3.6) yields the half-order FermiDirac function as follows:

$$
\begin{gathered}
F_{1 / 2}(\xi)=\frac{2}{3} \xi^{3 / 2}+\frac{\sqrt{\pi}}{2} \sum_{p=1}^{\infty} \frac{1}{p^{3 / 2}}\left[e^{p \xi} \operatorname{Erfc}(\sqrt{p \xi})\right. \\
-\frac{1}{\sqrt{2}} e^{2 p \xi} \operatorname{Erfc}(\sqrt{2 p \xi})+e^{-p \xi} \operatorname{Erfi}(\sqrt{p \xi}) \\
\left.-\frac{1}{\sqrt{2}} e^{-2 p \xi} \operatorname{Erfi}(\sqrt{2 p \xi})\right] .
\end{gathered}
$$

Equation (E.3.9) can easily be checked by the application of (8). In doing so, one obtains [1]

$$
\begin{aligned}
& F_{1 / 2}(\xi)=\frac{2}{3} \xi^{3 / 2}-\frac{\sqrt{\pi}}{2} \sum_{p=1}^{\infty} \frac{(-1)^{p}}{p^{3 / 2}} \\
& \cdot\left[e^{p \xi} \operatorname{Erfc}(\sqrt{p \xi})+e^{-p \xi} \operatorname{Erfi}(\sqrt{p \xi})\right] .
\end{aligned}
$$

Equations (E.3.9) and (E.3.10) yield different-looking results but are actually identities. Either (E.3.9) or (E.3.10) can be used to define the half-order Fermi-Dirac function. Alternatively, (5) and (6) could be used to analytically evaluate the half-order Fermi-Dirac function or the half-order BoseEinstein function, respectively, without employing contour integration.

3.4. Example 4. As a final example, and the most difficult of the four examples discussed in this article, the authors will analytically evaluate the incomplete half-order Fermi-Dirac function [27] defined as follows:

$$
F_{1 / 2}(\xi, u)=\int_{0}^{u} \frac{\sqrt{x}}{1+e^{x-\xi}} d x
$$

valid $\forall \xi \in \mathbb{R}$ and $\forall u \in \mathbb{R} \geq 0$. Rewrite (E.4.1) as follows:

$$
\begin{aligned}
F_{1 / 2}(\xi, u) & =\int_{0}^{\infty} \frac{\sqrt{x}}{1+e^{x-\xi}} d x-\int_{u}^{\infty} \frac{\sqrt{x}}{1+e^{x-\xi}} d x \\
& =F_{1 / 2}(\xi)-G_{1 / 2}(\xi, u) .
\end{aligned}
$$

To evaluate $G_{1 / 2}(\xi, u)$, let $y=x-u$ in the second integral of (E.4.2). The result is as follows:

$$
G_{1 / 2}(\xi, u)=\int_{0}^{\infty} \frac{\sqrt{y+u}}{1+e^{y-(\xi-u)}} d y .
$$

Some care must be taken when considering (E.4.4). There are three cases which need to be considered. These cases are as follows.

Case 1. Find $G_{1 / 2}(\xi, u) \forall \xi \in \mathbb{R} \geq 0, \forall u \in \mathbb{R} \leq \xi$.

Case 2. Find $G_{1 / 2}(\xi, u) \forall \xi \in \mathbb{R} \geq 0, \forall u \in \mathbb{R} \geq \xi$.

Case 3. Find $G_{1 / 2}(\xi, u) \forall \xi \in \mathbb{R} \leq 0, \forall u \in \mathbb{R} \geq 0$.

The authors will only analyze Case 1 since the other cases follow the same procedure. One can rewrite (E.4.2), employing (E.4.4), as follows:

$$
F_{1 / 2}(\xi, u)=F_{1 / 2}(\xi)-\int_{0}^{\infty} \frac{\sqrt{y+u}}{1+e^{y-(\xi-u)}} d y .
$$

An expression for the half-order Fermi-Dirac function is given by (E.3.10). Evaluate $G_{1 / 2}(\xi, u)$ using $(8)$ with $\mathbb{F}(s)$ given by

$$
\mathbb{F}(s)=\frac{2 \sqrt{s u}+\sqrt{\pi} e^{s u} \operatorname{Erfc}(\sqrt{s u})}{2 s^{3 / 2}} .
$$

Employing (E.4.5), (E.4.6), and (8) yields the following expression for $G_{1 / 2}(\xi, u)$ :

$$
\begin{aligned}
G_{1 / 2}(\xi, u)= & \frac{2}{3}\left(\xi^{3 / 2}-u^{3 / 2}\right) \\
& +\sqrt{\pi} \sum_{p=1}^{\infty}(-1)^{p} I(u, p),
\end{aligned}
$$

where

$$
\begin{aligned}
& I(u, p) \\
& \quad=\frac{1}{2 \pi i} \int_{\sigma-i \infty}^{\sigma+i \infty} \frac{2 \sqrt{s u / \pi}+e^{s u} \operatorname{Erfc}(\sqrt{s u})}{\sqrt{s}\left(s^{2}-p^{2}\right)} e^{(\xi-u) s} d s .
\end{aligned}
$$

Take note that $\xi$ in (8) has been replaced by $\xi-u$ in the evaluation of $G_{1 / 2}(\xi, u)$. This is true because the effective degeneracy parameter in (E.4.5) is $\xi-u$. Evaluate $I(u, p)$ by employing the Fermi-Dirac contour and substituting the result in (E.4.7). This yields the following:

$$
\begin{aligned}
& G_{1 / 2}(\xi, u)=\frac{2}{3}\left(\xi^{3 / 2}-u^{3 / 2}\right)+\sqrt{u} \ln \left[1+e^{-(\xi-u)}\right] \\
& -\frac{\sqrt{\pi}}{2} \sum_{p=1}^{\infty} \frac{(-1)^{p}}{p^{3 / 2}}\left\{e^{\xi p} \operatorname{Erfc}(\sqrt{p \xi})\right. \\
& \left.-e^{-\xi p}[\operatorname{Erfi}(\sqrt{u p})-\operatorname{Erfi}(\sqrt{\xi p})]\right\} .
\end{aligned}
$$


Finally, employing (E.3.10), (E.4.3), and (E.4.9) yields the analytical solution for the incomplete half-order Fermi-Dirac function valid $\forall \xi \in \mathbb{R} \geq 0$ and $\forall u \in \mathbb{R} \leq \xi$.

$$
\begin{aligned}
F_{1 / 2}(\xi, u)= & \frac{2}{3} u^{3 / 2}-\sqrt{u} \ln \left[1+e^{-(\xi-u)}\right] \\
& -\frac{\sqrt{\pi}}{2} \sum_{p=1}^{\infty} \frac{(-1)^{p}}{p^{3 / 2}} e^{-\xi p} \operatorname{Erfi}(\sqrt{u p}) .
\end{aligned}
$$

The result given by (E.4.10) is easily checked numerically by employing Mathematica [23]. Also, it is in complete numerical agreement with that published by Keller and Fenwick [27]. The authors believe that (E.4.10) exists nowhere else in the published literature. It is a simple matter to repeat the process to analytically evaluate the incomplete half-order Bose-Einstein function.

We leave it to the interested reader to verify that the incomplete half-order Fermi-Dirac functions for Cases 2 and 3 , respectively, are as follows:

$$
\begin{aligned}
& F_{1 / 2}(\xi, u)=\frac{2}{3} \xi^{3 / 2}-\sqrt{u} \ln \left[1+e^{-(u-\xi)}\right]-\frac{\sqrt{\pi}}{2} \\
& \cdot \sum_{p=1}^{\infty} \frac{(-1)^{p}}{p^{3 / 2}}\left\{e^{p \xi}[\operatorname{Erf}(\sqrt{u p})-\operatorname{Erf}(\sqrt{\xi p})]\right. \\
& \left.+e^{-p \xi} \operatorname{Erfi}(\sqrt{p \xi})\right\}, \\
& F_{1 / 2}(\xi, u)=-\sqrt{u} \ln \left[1+e^{-(u+|\xi|)}\right]-\frac{\sqrt{\pi}}{2} \\
& \cdot \sum_{p=1}^{\infty} \frac{(-1)^{p}}{p^{3 / 2}} e^{-p|\xi|} \operatorname{Erf}(\sqrt{u p}) .
\end{aligned}
$$

\section{Remarks and Conclusion}

This article illustrates the utility of real convolution for analytically evaluating the Fermi-Dirac-type and Bose-Einsteintype functions. These functions occupy a very important role in statistical mechanics, quantum statistical mechanics, solid-state physics, and others. The chosen technique is a generalization of the method developed by the authors [1]. A few examples are used to illustrate the efficacy of the method. In particular, the authors chose to look at the wellknown half-order Fermi-Dirac and half-order Bose-Einstein functions because of their importance in a number of areas of physics and engineering. However, the method developed in this paper is quite general and may be employed to analytically evaluate integrals given by (1) or (2) with an arbitrary $f(x)$. Of course, the functional form of $f(x)$ may be such that the integrals in (1) or (2) will be very difficult for analytical evaluation.

It is emphasized that one may choose to directly integrate the Fermi-Dirac-type or the Bose-Einstein-type functions by employing a direct method as exhibited by (5) or (6), or by contour integration as exhibited by (8) or (9). The method that is chosen lies with the discretion of the practitioner. However, the application of real convolution and contour integration was the main focus of the article because the
Fermi-Dirac-type functions and Bose-Einstein-type functions perfectly fit the form of real-convolution integrals.

Although this article focused on analytical solutions, a few comments should be made concerning numerical analysis. For example, a recent article written by Mohankumar and Natarajan [28] gives a very accurate method for numerically computing the Generalized Fermi-Dirac integrals. Also, recent articles by Changshi [29] and Fukushima [30] give excellent methods for numerically computing Fermi-Diractype integrals. There are many other published methods that work very well for numerically computing the Fermi-Diractype and Bose-Einstein-type integrals. The authors of this article employed the method developed by Chevillard and Revol [31, 32] and achieved promising numerical results. However, there will always be new and improved methods of numerical computation and the authors simply cannot predict with any certainty if the analytical solutions developed in this paper will be of greater or lesser utility than any other method when it comes to its use in numerical analysis. However, what the authors can state with certainty is that a very simple procedure has been developed for analytically evaluating Fermi-Dirac-type and Bose-Einsteintype integrals for a wide range of functions, $f(x)$. The technique employed for doing this may lead to new ideas for numerical formulations as well as asymptotic formulations and may provide new computational insight.

Analytical expressions can help aid in the development of approximate solutions $[33,34]$ as well as in the development of asymptotic solutions [35] which are used extensively for numerical computation. Often, a complete analytical solution can help to aid in the development of numerical algorithms as well as to give the researcher insight into how the parameters of an integral fit within the form of the solution. The authors believe that when an analytical solution can be found, even if mathematically complex, it can be of great utility. In fact, the analytical solutions developed in this article coupled with the methods developed by Chevillard and Revol [31,32] have aided the authors in the development of various algorithms for numerically computing the half-order Fermi-Dirac and half-order Bose-Einstein functions. Chevillard's method is based upon a set of algorithms for numerically computing error functions with great accuracy. The results, as stated earlier, appear to be quite promising. Further research is ongoing.

One must be cognizant, however, that analytical solutions are not necessarily used for high-precision numerical computations especially if the analytical solution is in the form of an infinite series. Often the series may be slower to converge for various values of its parameters, and this requires one to find a more efficient numerical or asymptotic solution. As stated earlier, many such algorithms currently exist in the literature.

One final point should be stressed. The method developed in this paper has recently been employed for analytically evaluating the Gauss-Fermi integral [36]. This integral was previously known to not have a complete analytical solution [37]. This is one more indication of the efficacy of the method for analytically evaluating Fermi-Dirac-type or Bose-Einstein-type integrals for a variety of functions, $f(x)$, illustrated in (1) and (2). 


\section{Appendix}

Any one of the half-order Fermi-Dirac or half-order BoseEinstein functions enables any other half-order Fermi-Dirac or half-order Bose-Einstein functions to be evaluated $\forall m \in$ $\mathbb{Z}^{\geq}$. This is illustrated in Dingle $[3,6]$ as follows:

$$
\begin{aligned}
\mathfrak{F}_{m-1 / 2}(\xi) & =\frac{1}{\Gamma(m+1 / 2)} \int_{0}^{\infty} \frac{x^{m-1 / 2}}{1+e^{x-\xi}} d x \\
& =\frac{1}{\Gamma(m+1 / 2)} F_{m-1 / 2}(\xi), \\
\mathfrak{B}_{m-1 / 2}(\xi) & =\frac{1}{\Gamma(m+1 / 2)} \int_{0}^{\infty} \frac{x^{m-1 / 2}}{-1+e^{x-\xi}} d x \\
& =\frac{1}{\Gamma(m+1 / 2)} B_{m-1 / 2}(\xi) .
\end{aligned}
$$

Employing (A.1), and the known recurrence relationships [3, 6] given by

$$
\begin{aligned}
& \mathfrak{\Im}_{m-1 / 2}(\xi)=\frac{d}{d \xi} \mathfrak{F}_{m+1 / 2}(\xi), \\
& \mathfrak{B}_{m-1 / 2}(\xi)=\frac{d}{d \xi} \mathfrak{B}_{m+1 / 2}(\xi),
\end{aligned}
$$

yields all the half-order Fermi-Dirac functions or half-order Bose-Einstein functions. For example, evaluate $\mathfrak{F}_{-1 / 2}(\xi)$ and $\mathfrak{F}_{3 / 2}(\xi)$ as follows:

$$
\begin{aligned}
& \mathfrak{F}_{-1 / 2}(\xi)=\frac{d}{d \xi} \mathfrak{\mho}_{1 / 2}(\xi) \\
& =2 \sqrt{\frac{\xi}{\pi}} \\
& \quad-\sum_{p=1}^{\infty} \frac{(-1)^{p}}{p^{1 / 2}}\left[e^{p \xi} \operatorname{Erfc}(\sqrt{p \xi})-e^{-p \xi} \operatorname{Erfi}(\sqrt{p \xi})\right], \\
& \mathfrak{\mho}_{3 / 2}(\xi)=\int \mathfrak{\mho}_{1 / 2}(\xi) d \xi \\
& =\frac{\pi \sqrt{\pi \xi}}{3}+\frac{8 \xi^{2}}{15} \sqrt{\frac{\xi}{\pi}} \\
& \quad-\sum_{p=1}^{\infty} \frac{(-1)^{p}}{p^{5 / 2}}\left[e^{p \xi} \operatorname{Erfc}(\sqrt{p \xi})-e^{-p \xi} \operatorname{Erfi}(\sqrt{p \xi})\right] .
\end{aligned}
$$

Likewise, evaluate $\mathfrak{B}_{-1 / 2}(\xi)$ and $\mathfrak{B}_{3 / 2}(\xi)$ as follows:

$$
\begin{aligned}
& \mathfrak{B}_{-1 / 2}(\xi)=\frac{d}{d \xi} \mathfrak{B}_{1 / 2}(\xi) \\
& =-2 \sqrt{\frac{\xi}{\pi}} \\
& \quad+\sum_{p=1}^{\infty} \frac{1}{p^{1 / 2}}\left[e^{p \xi} \operatorname{Erfc}(\sqrt{p \xi})-e^{-p \xi} \operatorname{Erfi}(\sqrt{p \xi})\right],
\end{aligned}
$$

$$
\begin{aligned}
& \mathfrak{B}_{3 / 2}(\xi)=\int \mathfrak{B}_{1 / 2}(\xi) \\
& =\frac{2 \pi \sqrt{\pi \xi}}{3}-\frac{8 \xi^{2}}{15} \sqrt{\frac{\xi}{\pi}} \\
& \quad+\sum_{p=1}^{\infty} \frac{1}{p^{5 / 2}}\left[e^{p \xi} \operatorname{Erfc}(\sqrt{p \xi})-e^{-p \xi} \operatorname{Erfi}(\sqrt{p \xi})\right] .
\end{aligned}
$$

All half-order Fermi-Dirac and half-order Bose-Einstein functions are easily evaluated using (A.1) and (A.2).

\section{Disclosure}

Jerry P. Selvaggi is a Former Visiting Associate Professor at SUNY New Paltz, New Paltz, NY 12561. Jerry A. Selvaggi is a Retired Professor at Gannon University, Erie, PA 16501.

\section{Conflicts of Interest}

The authors declare that there are no conflicts of interest regarding the publication of this paper.

\section{References}

[1] J. A. Selvaggi and J. P. Selvaggi, "The analytical evaluation of the half-order Fermi-Dirac integrals," Open Mathematics Journal, vol. 5, pp. 1-7, 2012.

[2] J. S. Blakemore, "Approximations for Fermi-Dirac integrals, especially the function $\mathrm{F}_{1 / 2}(\eta)$ used to describe electron density in a semiconductor," Solid-State Electronics, vol. 25, no. 11, pp. 1067-1076, 1982.

[3] R. B. Dingle, "The fermi-dirac integrals $\mathfrak{\Im}_{p}(\eta)=$ $(p !)^{-1} \int_{0}^{\infty} \varepsilon^{p}\left(e^{\varepsilon-\eta}+1\right)^{-1} d \varepsilon$, , Applied Scientific Research, vol. 6, no. 1, pp. 225-239, 1957.

[4] J. McDougall and E. C. Stoner, "The Computation of FermiDirac Functions," Philosophical Transactions of the Royal Society A: Mathematical, Physical \& Engineering Sciences, vol. 237, no. 773, pp. 67-104, 1938.

[5] J. Clunie, "On Bose-Einstein functions," Proceedings of the Physical Society. Section A, vol. 67, no. 7, article no. 308, pp. 632636, 1954.

[6] R. B. Dingle, "The Bose-Einstein integrals $\mathfrak{B}_{p}(\eta)=$ $(p !)^{-1} \int_{0}^{\infty} \varepsilon^{p}\left(e^{\varepsilon-\eta}-1\right)^{-1} d \varepsilon, "$ Applied Scientific Research, Section A, vol. 6, pp. 240-244, 1957.

[7] K. Huang, Statistical Mechanics, John Wiley \& Sons, Inc., New York, NY, USA, 2nd edition, 1963.

[8] L. E. Reichl, A Modern Course in Statistical Physics, Wiley-VCH, Berlin, Germany, 2nd edition, 2004.

[9] R. N. Hill, "A note on certain integrals which appear in the theory of the ideal quantum gases," American Journal of Physics, vol. 38, pp. 1440-1447, 1970.

[10] W. J. Cody and H. C. Thacher, "Rational Chebyshev approximations for fermi-dirac integrals of orders $-1 / 2,1 / 2$ and $3 / 2$," Mathematics of Computation, vol. 21, no. 97, pp. 30-40, 1967.

[11] T. M. Garoni, N. E. Frankel, and M. L. Glasser, "Complete asymptotic expansions of the Fermi-Dirac integrals $\mathfrak{J}_{p}(\eta)=$ $1 / \Gamma(p+1) \int_{0}^{\infty} \varepsilon^{p} /\left(1+e^{\varepsilon-\eta}\right) d \varepsilon, "$ Journal of Mathematical Physics, vol. 42, no. 4, pp. 1860-1868, 2001. 
[12] M. Goano, "Algorithm 745: Computation of the Complete and Incomplete Fermi-Dirac Integral," ACM Transactions on Mathematical Software, vol. 21, no. 3, pp. 221-232, 1995.

[13] M. Goano, "Series expansion of the Fermi-Dirac integral $F_{j}(x)$ over the entire domain of real $j$ and $x$," Solid-State Electronics, vol. 36, no. 2, pp. 217-221, 1993.

[14] A. J. Macleod, "Algorithm 779: Fermi-Dirac Functions of Order $-1 / 2,1 / 2,3 / 2,5 / 2$," ACM Transactions on Mathematical Software, vol. 24, no. 1, pp. 1-12, 1998.

[15] N. Mohankumar, "Two new series for the Fermi-Dirac integral," Computer Physics Communications, vol. 176, no. 11-12, pp. 665669, 2007.

[16] N. Mohankumar and A. Natarajan, "The accurate numerical evaluation of half-order Fermi-Dirac Integrals," Physica Status Solidi (b) - Basic Solid State Physics, vol. 188, no. 2, pp. 635-644, 1995.

[17] M. A. Chaudhry and A. Qadir, "Operator representation of Fermi-Dirac and Bose-Einstein integral functions with applications," International Journal of Mathematics and Mathematical Sciences, vol. 2007, Article ID 80515, 9 pages, 2007.

[18] E. W. Ng, C. J. Devine, and R. F. Tooper, "Chebyshev polynomial expansion of Bose-Einstein functions of orders 1 to $10^{*}$," Mathematics of Computation, vol. 23, pp. 639-643, 1969.

[19] D. Cvijovi, "New integral representations of the polylogarithm function," Proceedings of the Royal Society A Mathematical, Physical and Engineering Sciences, vol. 463, no. 2080, pp. 897905, 2007.

[20] M. H. Lee, "Polylogarithms and Riemann's $\zeta$ function," Physical Review E: Statistical, Nonlinear, and Soft Matter Physics, vol. 56, no. 4, pp. 3909-3912, 1997.

[21] M. H. Lee, "Polylogarithmic analysis of chemical potential and fluctuations in a $D$-dimensional free Fermi gas at low temperatures," Journal of Mathematical Physics, vol. 36, no. 3, pp. 1217-1231, 1995.

[22] M. D. Ulrich, W. F. Seng, and P. A. Barnes, "Solutions to the Fermi-Dirac Integrals in Semiconductor Physics Using Polylogarithms," Journal of Computational Electronics, vol. 1, no. 3, pp. 431-434, 2002.

[23] Wolfram Research, Inc., MATHEMATICA, version 11.2, Wolfram Research, Inc., Champaign, Ill, USA, 2017.

[24] I. N. Sneddon, Fourier Transforms, McGraw-Hill, New York, NY, USA, 1951.

[25] N. W. McLachlan, Complex Variable Theory \& Transform Calculus, Cambridge University Press, 2nd edition, 1955.

[26] B. van der Pol and H. Bremmer, Operational Calculus, Based on the Two-Sided Laplace Integral, Cambridge University Press, 1955.

[27] G. Keller and M. Fenwick, "Tabulation of the incomplete FermiDirac functions," The Astrophysical Journal, vol. 117, pp. 437-446, 1953.

[28] N. Mohankumar and A. Natarajan, "On the very accurate numerical evaluation of the generalized Fermi-Dirac integrals," Computer Physics Communications, vol. 207, pp. 193-201, 2017.

[29] L. Changshi, "More precise determination of work function based on Fermi-Dirac distribution and Fowler formula," Physica B: Condensed Matter, vol. 444, pp. 44-48, 2014.

[30] T. Fukushima, "Precise and fast computation of inverse FermiDirac integral of order $1 / 2$ by minimax rational function approximation," Applied Mathematics and Computation, vol. 259, pp. 698-707, 2015.
[31] S. Chevillard, "The functions erf and erfc computed with arbitrary precision and explicit error bounds," Information and Computation, vol. 216, pp. 72-95, 2012.

[32] S. Chevillard and N. Revol, "Computation of the error function erf in arbitrary precision with correct rounding," in Proceedings of the 8th Conference on Real Numbers and Computers, pp. 2736, 2008.

[33] O. N. Koroleva, A. V. Mazhukin, V. I. Mazhukin, and P. V. Breslavskiy, "Analytical Approximation of the Fermi-Dirac Integrals of Half-Integer and Integer Orders," Mathematical Models and Computer Simulations, vol. 9, pp. 383-389, 2017.

[34] F. J. Fernandez Velicia, "High-precision analytic approximations for the Fermi-Dirac functions by means of elementary functions," Physical Review A: Atomic, Molecular and Optical Physics, vol. 34, no. 5, pp. 4387-4395, 1986.

[35] J. Boersma and M. L. Glasser, "Asymptotic expansion of a class of Fermi-Dirac integrals," SIAM Journal on Mathematical Analysis, vol. 22, no. 3, pp. 810-820, 1991.

[36] J. P. Selvaggi, "Analytical evaluation of the charge carrier density of organic materials with a Gaussian density of states revisited," Journal of Computational Electronics, vol. 17, no. 1, pp. 61-67, 2017.

[37] G. Paasch and S. Scheinert, "Charge carrier density of organics with Gaussian density of states: Analytical approximation for the Gauss-Fermi integral," Journal of Applied Physics, vol. 107, no. 10, pp. 104501-1-104501-4, 2010. 


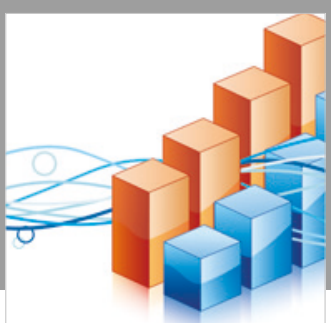

Advances in

Operations Research

\section{-n-m}
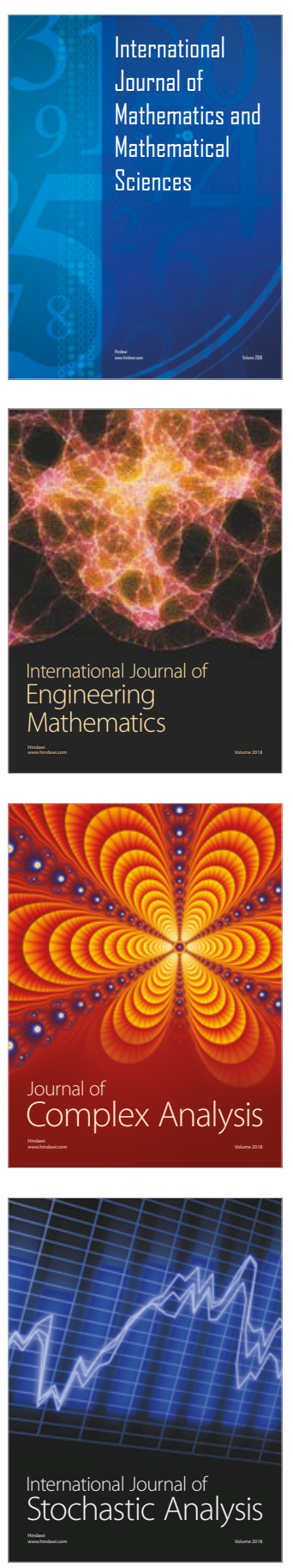
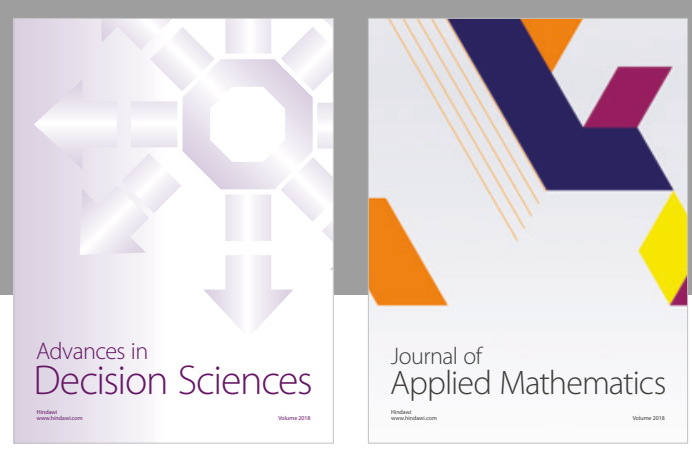

Journal of

Applied Mathematics
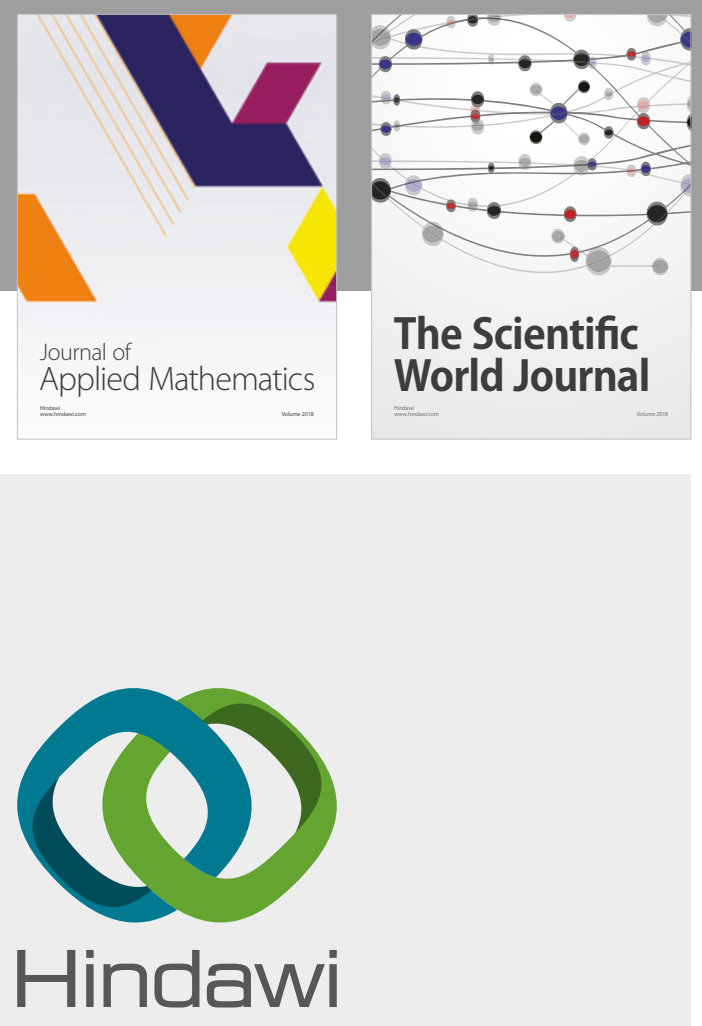

Submit your manuscripts at

www.hindawi.com

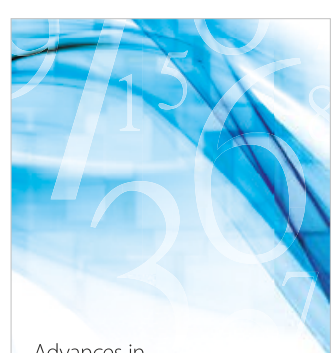

Advances in
Numerical Analysis
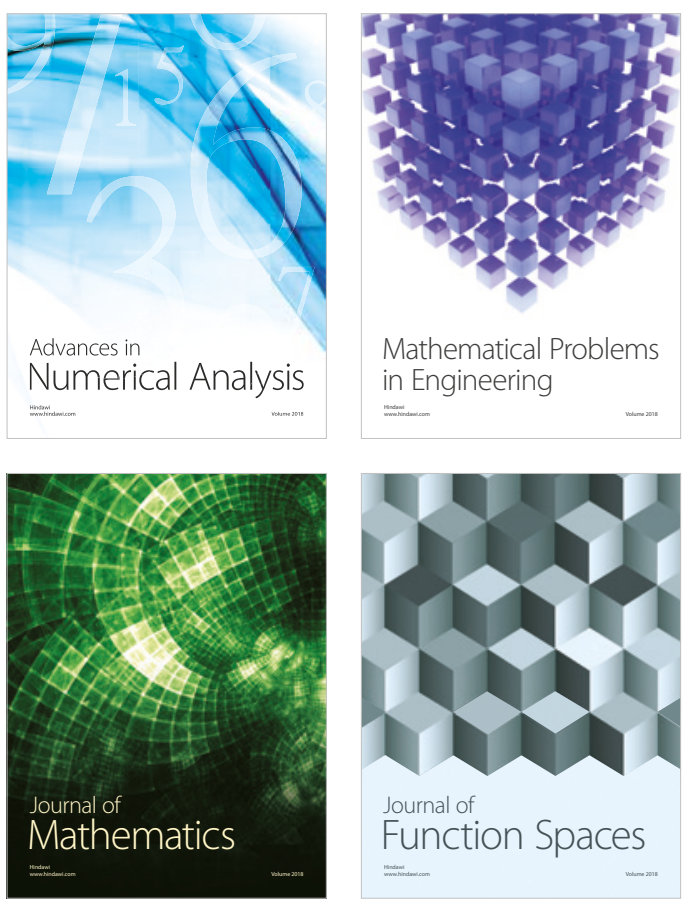

Mathematical Problems in Engineering

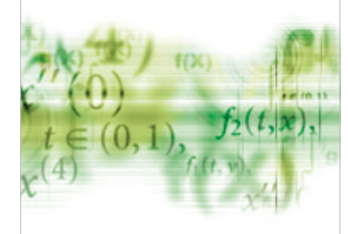

International Journal of

Differential Equations

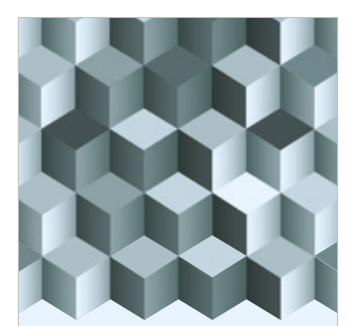

Journal of

Function Spaces

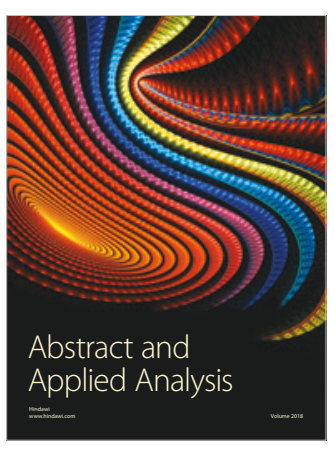

The Scientific

World Journal

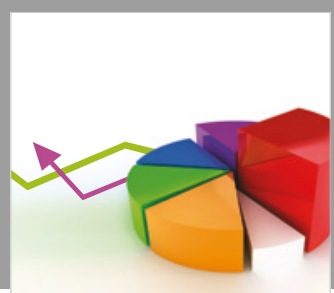

Journal of

Probability and Statistics
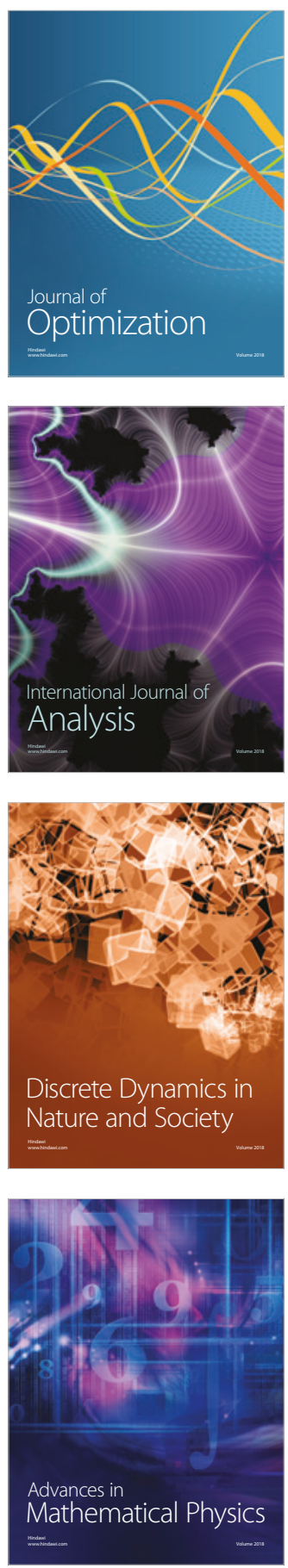